Regards sur l'économie allemande

Bulletin économique du CIRAC

$104 \mid 2012$

Varia

\title{
La TVA en France et en RFA : un révélateur des différences de politique fiscale
}

\section{Christophe Strassel}

\section{(2) OpenEdition}

\section{Journals}

Édition électronique

URL : http://journals.openedition.org/rea/4394

DOI : $10.4000 /$ rea.4394

ISBN : 978-2-8218-1286-4

ISSN : 1965-0787

\section{Éditeur}

CIRAC

\section{Édition imprimée}

Date de publication : 13 avril 2012

Pagination : 23-30

ISSN : 1156-8992

Référence électronique

Christophe Strassel, « La TVA en France et en RFA : un révélateur des différences de politique fiscale », Regards sur l'économie allemande [En ligne], 104 | avril 2012, mis en ligne le 01 avril 2014, consulté le 03 mai 2019. URL : http://journals.openedition.org/rea/4394 ; DOI : 10.4000/rea.4394 


\section{La TVA en France et en RFA : un révélateur des différences de politique fiscale}

\author{
Christophe Strassel
}

La TVA est un prélèvement symbolique à plusieurs égards. Créée en France au début des années 1950, elle est depuis 1967 le principal impôt ayant fait l'objet d'une harmonisation au niveau européen. Son efficacité en termes de rendement et sa relative neutralité économique en ont fait l'une des ressources fiscales principales des Etats membres de I'Union européenne. La TVA joue donc en Europe un rôle économique et budgétaire de premier plan.

Bien ancrée dans le paysage fiscal et économique des pays européens, la TVA continue pourtant de susciter des débats passionnés. Le contexte de la mondialisation a contribué à renforcer sa place dans les ressources fiscales. Elle repose en effet sur une assiette - la consommation - difficilement délocalisable. C'est pourquoi l'OCDE recommande régulièrement d'en augmenter le poids pour diminuer celui des impôts portant sur des assiettes plus mobiles : capital ou travail hautement qualifié. C'est ce qu'ont fait les économies du nord de l'Europe, comme la Suède ou le Danemark, en augmentant massivement leur taux de TVA dès les années 1990. En France, le débat s'est plutôt porté sur les vertus supposées de la TVA pour renforcer la compétitivité des entreprises: la "TVA sociale ", qui désigne la substitution de la TVA aux cotisations sociales pour le financement de l'Etat Providence, est évoquée depuis le début des années 1980. Pour autant, une telle évolution suscite bien des résistances, au regard notamment des conséquences d'une augmentation de la TVA sur le pouvoir d'achat des ménages les plus modestes.

L'une des curiosités du débat français sur la TVA est de mentionner régulièrement le "modèle allemand". La réussite à l'exportation de l'Allemagne est ainsi souvent rapprochée de sa politique en matière de TVA, et notamment du choix fait en 2007 par le gouvernement d'Angela Merkel d'augmenter de 3 points le taux de ce prélèvement. L'invocation du "modèle allemand " est devenue tellement courante en France que l'on peut se demander si elle est toujours pertinente. L'objet du présent article est, à travers l'exemple de la TVA, d'illustrer les différences de politique fiscale entre la France et l'Allemagne. II sera montré que derrière les convergences formelles se cachent en réalité des orientations, des priorités mais aussi des résultats très différents.

\section{Des régimes de TVA formellement proches}

L'harmonisation des règles d'assiette de la TVA a été considérée dès les années 1960 comme un complément nécessaire à la construction du marché commun. La chose n'allait pourtant pas de soi. En premier lieu parce que la situation des pays européens au regard de la TVA était très diversifiée dans les premières années de la construction européenne et demandait de profondes évolutions de la part de certains d'entre eux; en second lieu parce que des règles communes de taxation de la consommation ne sont pas indispensables au bon fonctionnement d'un marché commun. Les Etats-Unis connaissent ainsi des règles de taxation de la consommation différentes d'un Etat à l'autre sans que cela semble remettre en cause la circulation des biens et des services sur leur territoire.

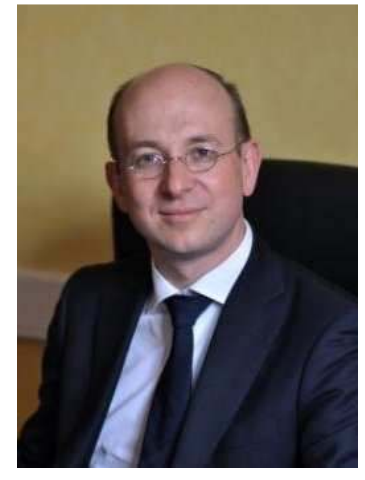

Christophe Strassel, économiste, professeur associé à l'ENS (rue d'Ulm)
Les règles d'assiette de la TVA relèvent du cadre communautaire 
Des marges de manœuvre nationales en matière de taux...

... à l'intérieur d'une fourchette

Rapprochement des taux entre la France et la RFA, ...
La première directive TVA a toutefois été adoptée au niveau communautaire en 1967. Elle a été suivie de nombreuses modifications qui ont été consolidées dans la " sixième directive TVA » du 17 mai 1977. Modernisé - quoiqu'à grandpeine en raison de la règle d'unanimité qui prévaut au sein de l'Union européenne en matière fiscale -, ce texte a été récemment refondu dans le cadre de la directive TVA du 28 novembre 2006. Les règles correspondantes ont été transposées dans les droits nationaux de l'ensemble des Etats membres: en France, elles figurent dans le Code général des impôts et en Allemagne, dans la « Loi sur la TVA » (Umsatzsteuergesetz).

La jurisprudence de la Cour de justice de l'UE ayant progressivement fait disparaître les divergences d'interprétation qui pouvaient subsister dans les législations nationales, il ne subsiste pas aujourd'hui de différence significative entre les pays européens concernant les règles d'assiette de la TVA.

Si le droit communautaire a imposé une harmonisation des règles d'assiette de la TVA dès 1967, la fixation des taux est longtemps restée une prérogative purement nationale. Cette autonomie des Etats peut se comprendre d'un point de vue économique : l'assiette de la TVA étant relativement peu mobile (un consommateur se déplace rarement dans un autre pays pour réaliser une transaction au seul motif que la TVA y serait à un niveau plus faible...), des différences de taux de TVA n'engendrent pas de distorsions économiques significatives. Seules les régions frontalières peuvent avoir à faire face à des phénomènes de concurrence par les taux de TVA. II n'est pas surprenant à cet égard que les revendications en faveur d'un taux de TVA réduit dans l'hôtellerie et la restauration aient souvent été portées par des professionnels proches de la frontière avec l'Espagne, pays dans lequel le taux de TVA applicable à ces activités a longtemps été plus faible qu'en France.

Après une première tentative dans les années 1980, un accord a finalement été trouvé pour fixer non pas un taux mais une fourchette de taux de TVA. La directive du 18 octobre 1992, dont les dispositions ont été reprises dans celle du 28 novembre 2006, prévoit ainsi deux grands types de dispositions :

- le taux normal de TVA est au minimum de $15 \%$; il n'existe pas de plafond, mais les Etats membres se sont engagés à respecter un plafond de $25 \%$, qui correspond au maximum observé notamment en Suède et au Danemark ;

- les taux réduits (deux au maximum) doivent être compris entre $5 \%$ et $15 \%$. Ils ne peuvent concerner que les biens et services énumérés dans l'annexe III de la directive de 2006 (notamment les biens alimentaires de première nécessité, les médicaments, les transports...).

Des dérogations sont en outre prévues permettant pour l'essentiel une taxation à un taux réduit d'activités qui, bien que ne figurant pas à l'annexe III, bénéficiaient d'un tel régime avant 1991 (ces taux dits " parking " ne bénéficient plus qu'à un nombre réduit de pays, par exemple pour le vin au Portugal ou certains biens énergétiques en Belgique). Des exceptions géographiques sont également prévues : elles concernent la Corse et les départements d'outre-mer qui bénéficient de taux spéciaux, ou encore, du côté allemand, l'île d'Helgoland et le territoire de Büsingen, exclus du champ de la TVA.

Dans ce contexte, les taux applicables en France et en Allemagne se sont fortement rapprochés. Le taux normal s'élève aujourd'hui à $19 \%$ en Allemagne contre $19,6 \%$ en France. La situation est la même concernant les taux réduits : $7 \%$ en Allemagne contre un double taux de $5,5 \%$ et $7 \%$ en France depuis 2012. Ces taux placent les deux pays à des niveaux inférieurs à la moyenne européenne qui était en 2011 de $20,52 \%$ pour le taux normal et de 8,46 \% pour le taux réduit (Cour des comptes, mars 2011).

Cette convergence franco-allemande concernant les taux de TVA est récente, et son sens doit être resitué dans l'évolution de long terme des prélèvements fiscaux au sein des deux pays. En 1970, la France appliquait un taux normal de TVA deux fois supérieur à celui de l'Allemagne : $23 \%$ contre $11 \%$. A cette 
époque, la France qui avait la première introduit la TVA dans son système de prélèvements obligatoires, sollicitait cet impôt bien plus que ses voisins. L'une des raisons de cette situation est la faiblesse récurrente de l'impôt sur le revenu en France : introduit en 1916, l'impôt sur le revenu français a toujours suscité des résistances culturelles et politiques importantes. II en a résulté une multiplication de dispositifs visant à en atténuer le poids - ce que l'on appelle aujourd'hui des " niches fiscales " - qui ont fortement diminué son rendement. De son côté, l'Allemagne a généralisé l'impôt sur le revenu en 1920, mais dans des conditions qui ont assuré d'emblée un rendement plus satisfaisant qu'en France. En 1950, la France tirait ainsi 45,5 \% de ses recettes fiscales d'impôts indirects contre $27,8 \%$ seulement en Allemagne (Atelier historique francoallemand, 2006).

L'invention de la TVA en France en 1954 doit donc être rapprochée de l'absence, à cette époque, d'un prélèvement moderne à fort rendement. Moins de vingt ans plus tard, lors de l'adoption de la première directive TVA, l'Allemagne conserve un système fiscal fondé en grande partie sur les impôts directs, alors que les impôts indirects sont plus développés en France.

Ensuite, un rapprochement graduel s'est opéré : en 1998, l'écart entre le taux normal français et celui de l'Allemagne n'est plus que de plus 5 points $(20,6 \%$ en France, $16 \%$ en Allemagne). Une bonne partie du rattrapage du taux normal allemand s'effectue ensuite à la faveur de deux hausses successives : un point en 1999 et trois points en 2007, alors que pendant la même période le taux applicable en France baisse d'un point en 2000. La convergence des taux entre la France et l'Allemagne est donc le résultat de deux dynamiques très différentes : partant d'un niveau très faible, l'Allemagne a progressivement augmenté le taux de la TVA; à l'inverse, la situation française fait apparaître une stagnation du taux applicable, qui n'est pas très différent aujourd'hui de ce qu'il était dans les années 1970. A cet égard, l'Allemagne est beaucoup plus représentative de la moyenne européenne que ne l'est la France : cette dernière est l'un des rares pays à n'avoir pas fait augmenter ses prélèvements au titre la TVA au cours des trois dernières décennies alors que l'Allemagne, comme la plupart des autres pays européens, mobilise de plus en plus cette ressource fiscale.

Ces dynamiques différentes de la TVA en France et en Allemagne sont sans doute, pour partie, liées à l'histoire fiscale des deux pays. Partant d'un niveau de taxation de la consommation nettement plus faible, l'Allemagne avait des marges de progression plus importantes que la France. Mais l'histoire n'explique pas tout. Nombreux sont les pays, notamment au nord de l'Europe, qui ont porté leurs taux de TVA à des niveaux bien supérieurs à ceux observés aujourd'hui en France. Plus qu'un décalage historique, les différences de progression de la TVA entre la France et l'Allemagne renvoient à des conceptions différentes de la politique fiscale dans les deux pays.

\section{Des différences profondes en matière de politique fiscale}

Trois types d'objectifs peuvent être assignés à un prélèvement fiscal : le rendement, l'intervention économique et la redistribution.

Le rendement constitue en pratique l'objectif premier de la fiscalité. II s'agit en effet de permettre le financement des politiques publiques à travers des ressources fournissant un produit élevé. La TVA réunit l'essentiel de ces qualités : elle est peu coûteuse à prélever puisque les entreprises assurent son recouvrement auprès des consommateurs et elle est à l'origine de ressources élevées et stables dans le temps.

L'intervention économique est un autre objectif possible des politiques fiscales. Cet objectif peut prendre plusieurs formes. II peut être orienté vers la neutralité économique. Dès lors, l'impôt optimal est celui qui modifie le moins possible les comportements des agents économiques (consommateurs, entreprises). La
... mais deux dynamiques très différentes
Les objectifs de
la fiscalité : ...
... rendement, ...

... intervention économique... 
... et redistribution

TVA : priorité au rendement en Allemagne, ...

... objectifs multiples et épars en France théorie associe le concept de neutralité avec des impôts à assiette large et à taux faible. Toutefois, les pouvoirs publics peuvent aussi rechercher dans la fiscalité un instrument permettant d'orienter les comportements économiques. La démarche est tout autre et l'impôt est alors mobilisé pour sa capacité à influencer les choix des consommateurs ou des entreprises. Entrent dans cette catégorie les impôts destinés à décourager les comportements comportant des externalités négatives: taxes environnementales, taxes sur l'alcool... A l'inverse, des avantages fiscaux ciblés peuvent avoir pour objet d'encourager des comportements que les pouvoirs publics considèrent comme bénéfiques : c'est le sens, par exemple, des dispositifs soutenant les dépenses de recherche et développement des entreprises.

Enfin, la fiscalité peut poursuivre un objectif de redistribution. Elle modifie alors la répartition initiale des richesses - revenus et patrimoine - telle qu'elle résulte du jeu du marché pour tendre vers une répartition considérée comme plus équitable. L'instrument fiscal privilégié dans cette fonction est la progressivité du barème, qui permet de taxer d'autant plus fortement les richesses que celles-ci sont importantes.

Part des recettes de TVA dans le total des recettes fiscales (en \%)

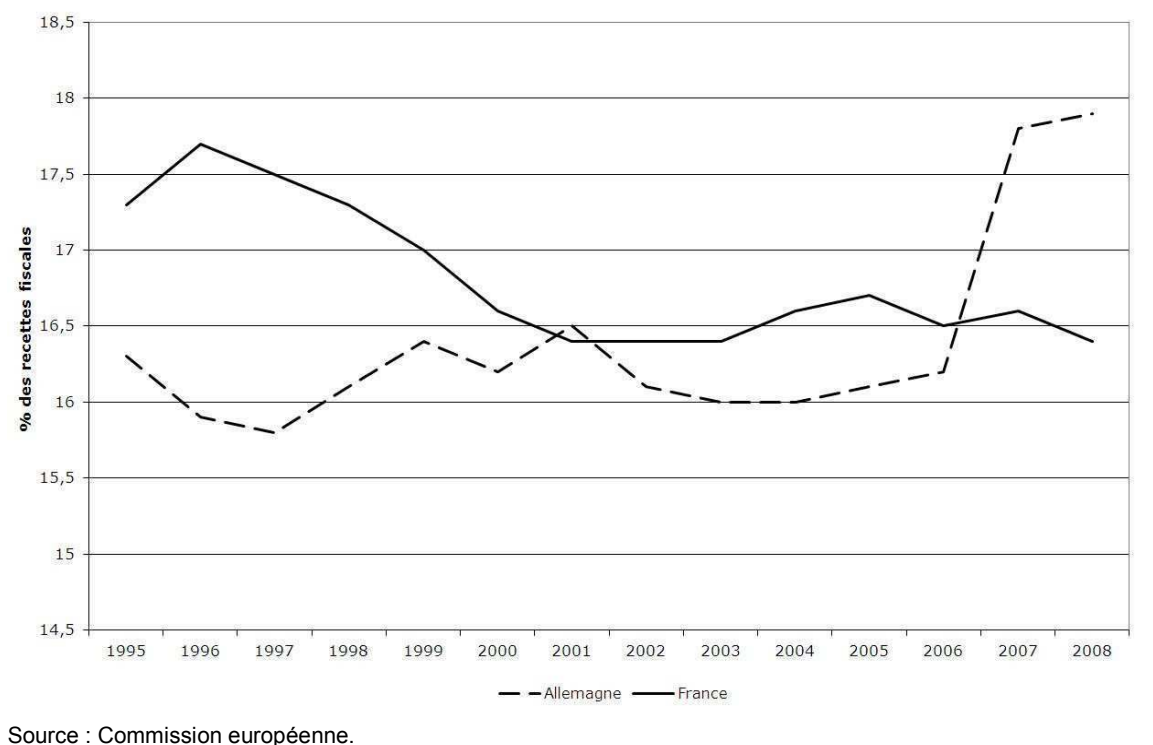

Comme tous les impôts, la TVA peut se voir assigner un ou plusieurs de ces objectifs. A cet égard, la situation qui prévaut en France est très différente de celle observée en Allemagne. Cette dernière privilégie clairement l'objectif de rendement concernant la TVA. La plupart des débats relatifs à cet impôt s'articulent en effet autour de ce thème. Ainsi, la hausse de la TVA entrée en vigueur au $1^{\text {er }}$ janvier 2007 a eu essentiellement pour objet d'augmenter les ressources des budgets publics. Sur les trois points d'augmentation de la TVA, deux ont été consacrés à la réduction des déficits publics, le troisième étant destiné à modérer la progression des cotisations d'assurance chômage. Par ailleurs, le débat lancé en 2010 sur la réduction du champ d'application du taux réduit s'est inscrit lui aussi dans une perspective d'amélioration du rendement.

A l'inverse, les objectifs assignés à la TVA en France apparaissent beaucoup plus diversifiés. Certes, la dimension de rendement de ce prélèvement, qui représente la moitié des ressources fiscales de l'Etat, n'est pas absente. Pour autant, elle n'a pas été prioritaire au cours des dernières années: la dernière hausse du taux normal de TVA date de 1995, et le second taux réduit entré en vigueur au $1^{\mathrm{er}}$ janvier 2012 n'a qu'un faible intérêt en termes de rendement en raison de son faible écart avec le taux réduit historique (7\% contre $5,5 \%)$. A l'inverse, le législateur français a assigné à la TVA des objectifs économiques 
ambitieux : lutte contre le travail clandestin (taux réduit de TVA pour les travaux à domicile depuis 1997), soutien sectoriel et créations d'emplois (taux réduit de TVA pour l'hôtellerie et la restauration depuis 2010).

Les résultats obtenus dans les deux pays au regard de ces objectifs sont assez contrastés. En termes de rendement, les différences sont très nettes : alors que la part de la TVA dans le total des ressources fiscales allemandes était historiquement inférieure à celle observée en France, cette part est devenue nettement supérieure à partir de 2007.

En termes de redistribution, les résultats sont également plus favorables en Allemagne qu'en France. Certes, la TVA est anti-redistributrice dans les deux pays : la part des dépenses consacrées à la TVA est plus élevée pour les ménages modestes que pour les ménages les plus aisés. Ce phénomène provient de ce que les ménages les plus modestes affectent la totalité de leurs revenus à la consommation - grevée par la TVA -, alors que les ménages les plus aisés en épargnent une partie - soustraite, donc à la TVA. La multiplication des taux réduits en France pourrait toutefois laisser à penser que ces aspects antiredistributifs pourraient y être moins prononcés. Dans la réalité, il n'en est rien : les $10 \%$ des ménages français les plus modestes consacrent $11,5 \%$ de leurs revenus à la TVA, contre $10,3 \%$ en Allemagne. A l'autre extrémité de l'échelle des revenus, les $10 \%$ des ménages les plus aisés consacrent une part identique de leurs revenus à la TVA dans les deux pays $(5,9 \%$ en France et en Allemagne).

Part du revenu des ménages consacré à la TVA en France et en Allemagne

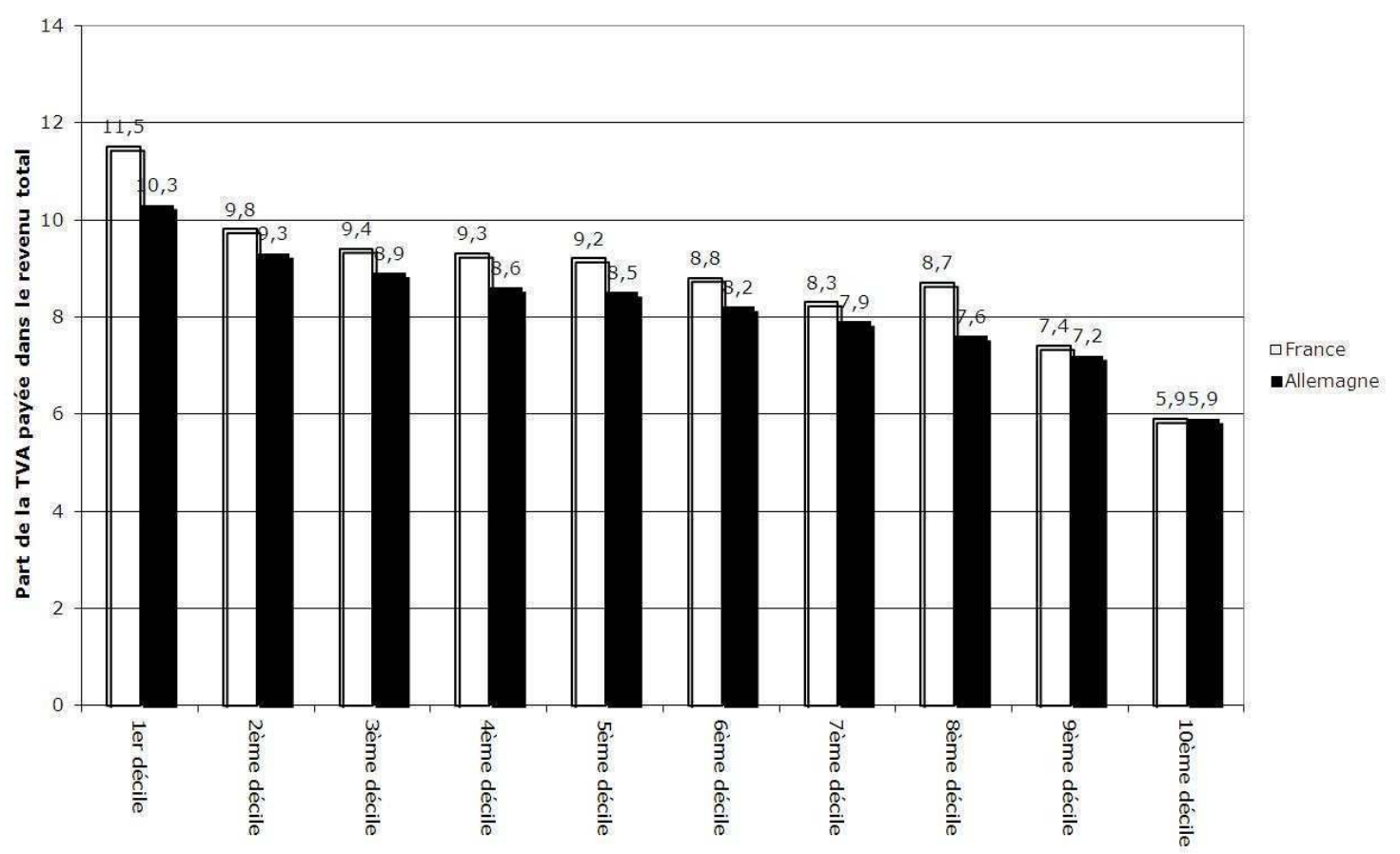

Source : Secrétariat d'Etat chargé de la prospective et de l'évaluation des politiques publiques, 2007.

Enfin, les résultats économiques obtenus par les baisses ciblées de taux de TVA en France restent difficilement mesurables : le gain en emploi serait compris entre 30000 et 50000 dans le secteur du bâtiment (soit un coût unitaire par emploi créé de 75000 à $135000 €$ ); il serait encore plus incertain dans le secteur de l'hôtellerie-café-restauration.

Une autre différence d'orientation des systèmes fiscaux français et allemand réside dans le nombre de taux utilisés : deux en Allemagne (taux normal et taux réduit) contre neuf en France (taux normal, deux taux réduits, un taux hyperréduit, cinq taux dérogatoires pour les départements d'outre-mer).
Rendement supérieur de la TVA en Allemagne...

... et redistribution plus prononcée
Un système de taux de TVA plus lisible et plus neutre en Allemagne 
Une conception différente du rôle économique de l'impôt

Deux approches du débat sur la compétitivité fiscale
II résulte de cette situation une grande complexité du système français, qui s'accompagne d'une moindre stabilité dans le temps. En effet, le recours plus large aux taux réduit s'est accompagné de modifications nombreuses de son périmètre d'application au cours des quinze dernières années : les travaux dans les locaux d'habitation sont passés au taux réduit en 1997 alors que le secteur de l'hôtellerie-cafés-restaurants a fait de même en 2010 ; l'introduction d'un second taux réduit de $7 \%$ en 2012 a encore complexifié le paysage en traçant une frontière difficile à appréhender entre les deux taux réduits.

Le dispositif allemand apparaît, par contraste, nettement plus stable et plus lisible. Certes, l'hôtellerie a bénéficié du passage au taux réduit lorsque cela a été rendu possible par la modification de la directive européenne, mais les modifications du champ d'application du taux réduit ne sont pas allées plus loin. Surtout, l'orientation qui prévaut désormais consiste à soumettre un nombre toujours plus restreint de biens et de services au taux réduit, afin de réserver ce dernier aux seuls produits de première nécessité.

Au-delà des questions de lisibilité et de stabilité, cette différence d'approche de la TVA permet de mettre en lumière une conception différente du rôle économique de l'impôt en France et en Allemagne. Conçue dans notre pays comme un instrument permettant d'orienter les comportements économiques, la TVA est utilisée de façon plus neutre en Allemagne. Cette différence renvoie à des traditions économiques très différentes, marquées en France par l'interventionnisme public et en Allemagne, par le souci d'organiser un cadre institutionnel stable et neutre économiquement, en droite ligne avec les conceptions héritées de l'ordolibéralisme.

La troisième différence marquante concerne la structuration du débat sur la compétitivité fiscale, qui s'est focalisé en grande partie autour du thème de la TVA sociale en France, alors que les progrès à cet égard ont surtout été recherchés, en Allemagne, sur le terrain de l'imposition des sociétés.

Les économies les plus ouvertes de l'UE sont souvent celles qui ont recours aux taux de TVA les plus élevés. Les pays les plus emblématiques de cette tendance sont la Suède et le Danemark qui ont porté leur taux normal de TVA à $25 \%$ dès le début des années 1990. L'Irlande, la Belgique et une grande partie des pays d'Europe centrale et orientale se caractérisent également par des taux de TVA élevés qui coexistent avec une forte ouverture aux échanges internationaux.

Taux d'ouverture et taux normal de TVA

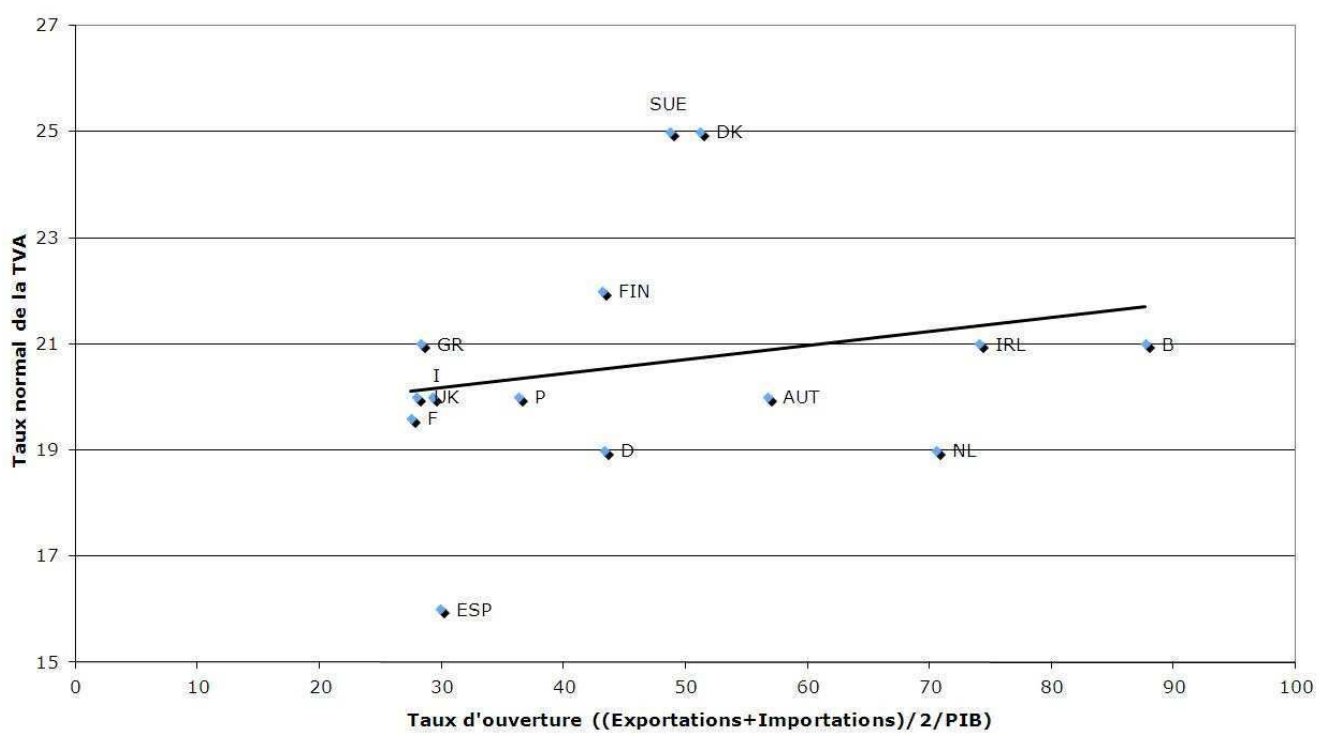

Source : chiffres Commission européenne (TVA) et OCDE (taux d'ouverture) 
Cette tendance peut s'expliquer par le fait que la TVA est généralement considérée comme un impôt dont les effets restent cantonnés au territoire national, et n'a que de faibles effets sur la localisation du travail et du capital. II est donc logique que les pays les plus ouverts aux biens et services étrangers, mais aussi aux mouvements de capitaux, soient ceux qui aient mis en œuvre les politiques fiscales privilégiant le plus la taxation de la consommation.

Au sein de I'UE, si la RFA a une économie plus ouverte que les autres pays de taille comparable (France, Italie, Royaume-Uni), elle reste proche de ces derniers pour ce qui est du niveau du taux normal de la TVA. Ni la France ni l'Allemagne n'ont donc fait un recours massif à la TVA comme instrument de compétitivité afin de faire baisser les prélèvements pesant, par exemple, sur le travail.

On entend généralement par "TVA sociale » la mesure par laquelle une hausse de la TVA compense une baisse de cotisations sociales pour financer une partie des dépenses de protection sociale. L'effet attendu de cette mesure est de conduire à une réduction du coût du travail dont il résulterait une amélioration de la compétitivité-prix des producteurs nationaux par rapport à leurs concurrents étrangers.

Celle-ci s'observerait à la fois :

- sur leur marché intérieur, dans la mesure où l'augmentation de la TVA frappe à la fois les biens et services produits localement et ceux qui sont importés. Les producteurs étrangers n'ayant pas bénéficié de la baisse des cotisations, ils voient leur situation se dégrader par rapport aux producteurs nationaux ;

- à l'exportation, dans la mesure où les entreprises exportatrices bénéficient de la baisse des cotisations sans avoir à subir la hausse de la TVA.

La réussite d'une telle opération dépend donc de la plus ou moins grande efficacité du report dans les prix hors taxes de la baisse du coût du travail ; en d'autres termes, l'augmentation des marges des entreprises ne doit pas absorber la totalité de la baisse des cotisations. Il convient également de noter que ce type de mesure est susceptible d'avoir des effets défavorables:

- en termes de croissance économique : la hausse de la TVA produit une augmentation plus ou moins prononcée des prix qui est de nature à freiner l'évolution de la consommation ;

- en termes de redistribution : la part de la TVA dans le revenu des ménages les plus modestes est supérieure à celle observée dans les ménages les plus favorisés; une augmentation de la TVA affecte donc davantage les catégories les ménages dont les revenus sont les plus modestes.

Evoquée dès le début des années 1980, notamment par les milieux patronaux, cette mesure est devenue un point de fixation du débat fiscal français. Le plus étonnant est de voir fréquemment mis en avant le " modèle allemand " dans ce domaine.

Dans la réalité, l'Allemagne n'a pas eu recours de manière significative à une mesure de type "TVA sociale ». En effet, les deux tiers de l'augmentation de la TVA mise en œuvre en Allemagne en 2007 ont été consacrés à la réduction du déficit budgétaire pour un montant de 16 milliards $€$. Le tiers restant a bien été affecté à une baisse des cotisations sociales: les cotisations d'assurance chômage sont ainsi passées de 6,5\% à 4,2\%, une partie de cette baisse ayant été financée par ailleurs, par une contribution de l'Agence fédérale pour l'emploi. Mais une telle baisse n'apparaît pas suffisamment forte pour avoir joué significativement sur la compétitivité de l'économie allemande. L'amélioration de cette dernière provient essentiellement de la faible progression des coûts salariaux depuis le début des années 2000 , à contre-courant des autres pays européens.

En définitive, la France a mis la TVA au centre du débat sur la compétitivité sans y avoir recours ; l'Allemagne, de son côté, a faiblement utilisé ce levier sans en avoir fait un objet de débat politique.
Mais ni la France ni la RFA ne recourent massivement à la TVA comme instrument de compétitivité

Avantages et désavantages de la " TVA sociale »

En RFA, la hausse de la TVA ne visait pas prioritairement la compétitivité 
C'est l'objectif de la fiscalité des sociétés
Si le débat français sur la compétitivité fiscale s'est porté sur la question de la TVA sociale, le débat allemand a plutôt été relatif à la fiscalité des sociétés. La prise de conscience, au début des années 1990, d'une crise de compétitivité du Standort Deutschland, a été à l'origine d'une baisse très prononcée de l'imposition des bénéfices à la fin de la décennie (qui prend en Allemagne la double forme de l'impôt sur les sociétés - Körperschaftsteuer - et de la taxe professionnelle destinée aux communes - Gewerbesteuer).

Taux nominal et taux effectif moyen (EATR ${ }^{1)}$ ) d'imposition des bénéfices en Allemagne ${ }^{2)}$ et en France (en \%)

\begin{tabular}{|c|c|c|c|c|c|c|c|c|}
\hline & \multicolumn{2}{|l|}{1998} & \multicolumn{2}{|l|}{2001} & \multicolumn{2}{|l|}{2005} & \multicolumn{2}{|l|}{2009} \\
\hline & Taux nominal & EATR & Taux nominal & EATR & Taux nominal & EATR & Taux nominal & EATR \\
\hline Allemagne & 56,5 & 41,2 & 39,4 & 35,8 & 39,4 & 35,8 & 31 & 28 \\
\hline France & 41,7 & 39,8 & 36,4 & 35,8 & 34,9 & 34,8 & 34,4 & 34,6 \\
\hline $\begin{array}{l}\text { Différence } \\
\text { Allemagne - France } \\
\text { (points de pourcentage) }\end{array}$ & 14,8 & 1,4 & 3,0 & 0,0 & 4,5 & 1,0 & $-3,4$ & $-6,6$ \\
\hline
\end{tabular}

Source : Zentrum für Europäische Wirtschaftsforschung. 1) Le taux effectif moyen d'imposition (EATR) reflète la réduction de la valeur actualisée nette d'un investissement rentable provoquée par l'impôt (un investissement rentable étant celui qui a un taux de rendement supérieur au coût du capital). Cet indicateur est particulièrement utile pour évaluer l'impact de la localisation d'un investissement sur son rendement après impôt. L'EATR est donc un indicateur d'attractivité. 2) Pour l'Allemagne, ce taux tient compte à la fois de l'impôt sur les sociétés et de la taxe professionnelle, dont les assiettes sont proches.

Elle est plus attractive en RFA
L'évolution des taux nominaux comme celle des taux effectifs montre une inversion de situation au cours des quinze dernières années entre la France et l'Allemagne: alors que la France avait, en termes d'imposition des bénéfices, une situation plus favorable que l'Allemagne à la fin des années 1990, la situation s'est progressivement inversée et fait désormais apparaître une attractivité nettement plus grande du côté allemand.

L'ÉTUde de L'Évolution RÉCENTE de LA TVA en France et en AlLEMAGne permet de mettre en évidence des différences importantes de politique fiscale - et même de philosophie fiscale - entre les deux pays. Alors que l'Allemagne a spécialisé la TVA sur un objectif de rendement, la France lui a assigné une multiplicité d'objectifs, parfois contradictoires.

Les résultats sont sans appel. Si l'invocation récurrente du « modèle allemand » comme référence pour la politique économique française est parfois mal fondée, tel n'est pas le cas en matière de TVA. Plus efficace en termes de rendement, moins anti-redistributive, la TVA allemande permet de réserver d'autres instruments - probablement plus efficaces que la TVA - aux objectifs d'emploi et de compétitivité économique.

\section{Indications bibliographiques}

- Atelier historique fRANCo-ALlemand, Finances publiques et administrations publiques - les ministères des finances et de l'économie français et allemand, Paris, 8 mars 2006 ; document téléchargeable sur : http://www.cirac.u-cergy.fr/cirac_forum/atelier_franco-allemand.pdf

-AUJeAn M. ET LoRENZI J.-H. (dir.), Fiscalité et croissānce, PUF, 2011

-Bourgeois I., « Réforme fiscale allemande : la lente construction des équilibres d'Helmut Kohl à Gerhard Schröder », Regards sur l'économie allemande, n 54/2001

- Commission EUROPÉENNE, Taux de TVA appliqués dans les Etats membres de l'Union européenne, situation au $1^{\text {er }}$ janvier 2012; document téléchargeable sur: http://ec. europa.eu/taxation_customs/resources/documents/taxation/vat/how_vat_works/rates/vat_rates_fr. pdf

-CONSEIL DES IMPÔTS, La TVA, XIXème rapport au président de la République, juin 2001

- CoUR DES COMPTES, Rapport sur les prélèvements fiscaux et sociaux en France et en Allemagne, mars 2011

-Deutscher Bundestag, Bericht der Bundesregierung zur Zukunftssicherung des Standortes Deutschland, BT-Drucksache 12/5620, 3 septembre 1993

-OCDE, Tax and Economic Growth, Economics Department Working Paper n 620, juillet 2008. 


\section{Actualité économique}

\section{Ð ÉNERGIE : la bronca des milieux économiques}

"Le chaos de cette nouvelle politique énergétique menace l'emploi!". C'est sous ce titre que le quotidien BILD publiait le 3 avril 2012 une interview de Hans-Dietrich Driftmann. Dans cet entretien, le président de la Fédération des $\mathrm{CCl}$ allemandes (DIHK) résumait entre autres les principaux griefs des milieux économiques allemands envers les pouvoirs politiques. La compétitivité allemande dépend en effet largement du succès de la mise en œuvre de cette nouvelle politique décidée à la suite de l'avarie du réacteur de Fukushima, et plus encore de sa rapidité. Or « le pire, c'est que, dans ce changement de politique, rien n'est clair - sauf son objectif [i.e. la sortie du nucléaire d'ici 2022 (voir REA 101/2011) ; IB]. Si nous n'avançons pas assez vite dans le développement des infrastructures, cela nous coûtera croissance et emplois ».

Et de fait, à part la fermeture de quelques centrales nucléaires et la réduction des subventions au secteur du photovoltaïque, peu d'éléments nouveaux sont intervenus depuis un an. Les réseaux de transport entre le nord de l'Allemagne où se concentre la production d'électricité éolienne et le sud, où se concentre la consommation industrielle, tardent à être modernisés, et le risque de black-out qui inquiète toujours l'autorité de régulation des réseaux (Bundesnetzagentur) reste présent. La situation est tendue au point que même le très sérieux quotidien Frankfurter Allgemeine Zeitung publiait le 16 mars un long papier d'humeur intitulé "Tous le pensent, personne ne le dit : la nouvelle politique énergétique est un échec ". Pour toutes les critiques actuellement formulées, le cœur du problème réside dans les sous-capacités en matière d'infrastructures et la lenteur de leur extension.

De fait, il n'y a pas en Allemagne une politique énergétique d'un seul tenant, mais au moins 17, étant donné la répartition des compétences afférentes entre plusieurs ministères à l'échelon fédéral, et l'autonomie des Länder qui définissent chacun sa propre politique de sortie du nucléaire et de promotion des renouvelables. Et, selon l'obédience de leurs gouvernements, ils se livrent à une véritable surenchère dans le mieux-disant écologique et proposent chacun son modèle. Restent les épineuses questions du stockage de l'électricité (source de conflits entre les impératifs de protection de la nature et ceux de compétitivité industrielle) et de l'aménagement des réseaux de transport qui soulève plusieurs problèmes : durée des procédures d'autorisation, opposition des riverains (syndrome du nimby, particulièrement prononcé outre-Rhin depuis les manifestations contre l'extension de la gare de Stuttgart: "Stuttgart 21 ») ou responsabilités peu claires en matière de financement public...

Pour les industriels, cette "cacophonie » (F.A.Z., 05-04-2012) et l'état prolongé d'incertitude qui s'ensuit et qui interdit toute planification, sont dramatiques. S'ils savent s'accommoder des changements de cap politiques, ils ne redoutent rien de plus que l'absence de prévisibilité.

Et dès lors, depuis quelques mois, les critiques des milieux économiques se multiplient. D'abord très convenues, voire diplomatiques, comme les divers catalogues de mesures concrètes proposées par la Fédération des $\mathrm{CCl}$ allemandes (DIHK) ou la Fédération de l'industrie chimique $(\mathrm{VCl})$, ou encore cette prise de position de la Fédération de l'industrie BDI en date du 14 décembre 2011, par laquelle son viceprésident appelle les pouvoirs publics à " ménager le tournant énergétique de manière à ce que la compétitivité mondiale de l'Allemagne n'en souffre pas et que soit préservé l'emploi industriel ". Mais au fur et à mesure que perdurent les incertitudes, et dans le contexte médiatique du premier anniversaire de la sortie du nucléaire, le ton se fait plus pressant.

C'est ainsi que, le 3 avril, Klaus Engel, président de la Fédération VCl et PdG du groupe Evonik, publiait dans le quotidien Handelsblatt une tribune libre: "Pas comme ça! ॥. II résume lui aussi avec force les griefs de ses homologues et s'insurge : " aucun investisseur n'est prêt à investir durablement des capitaux dans un marché aussi politisé et dont les règles sont susceptibles de changer à quelques mois d'intervalle ". Mais comme toujours en Allemagne, sa critique se veut avant
La question des infrastructures n'est pas résolue

Une « cacophonie " de politiques

Des critiques de plus en plus pressantes...

... et un appel à la co-décision 